\section{A tool to guide the process of integrating health system responses to public health problems}

\author{
Tilahun Nigatu Haregu, \\ Geoffrey Setswe, ${ }^{2}$ Jullian Elliott, ${ }^{3}$ \\ Brian Oldenburg ${ }^{4}$
}

${ }^{1}$ African Population and Health Research Center, Nairobi, Kenya; ${ }^{2}$ Human Sciences

Research Council, Pretoria, South Africa; ${ }^{3}$ Burnet institute, Melbourne; ${ }^{4}$ University of Melbourne, Australia

\section{Abstract}

An integrated model of health system responses to public health problems is considered to be the most preferable approach. Accordingly, there are several models that stipulate what an integrated architecture should look like. However, tools that can guide the overall process of integration are lacking. This tool is designed to guide the entire process of integration of health system responses to major public health problems. It is developed by taking into account the contexts of health systems of developing countries and the emergence of double-burden of chronic diseases in these settings. Chronic diseases - HIV/AIDS and NCDs - represented the evidence base for the development of the model. System level horizontal integration of health system responses were considered in the development of this tool.

\section{Introduction}

The tool presented here is based on the Analysis - Synthesis - Action continuum. It considers integration as a spiral rather than a linear process. The potential users of this tool are health policy makers, health care managers and health policy and systems researchers. These users may use this tool out of sequence based on their contexts and needs. As this tool is generic in its nature, users should adapt it to their own health system context, public health problems, and responses considered for integration.

This tool was developed based on an action model of integration presented elsewhere. ${ }^{1-5}$ It builds upon the best available evidence and it combines theoretical, empirical and practical evidence. It is worth noting that there are several other models that address the different components of this tool. ${ }^{6-10}$ This tool presents a unique consolidation of the translation of these models in a form of a guiding tool along with essential new elements. The contents of this tool are conceptually validated and were enriched using inputs from expert consultations.

This tool is divided into five major sections: i) analysing the connections between problems; ii) examining similarities between responses; iii) scanning the environment for integration; iv) repackaging evidence for communication; v) managing integration.

\section{Analysing the connections between the problems}

\section{Convergence between the prob- lems}

Understanding population level (epidemiological) overlap between the distributions of two public health problems is important to inform overall policy approaches that address the problems. Considering the socio-ecological model, epidemiological overlap between two diseases has three dimensions: population groups (segments of the population based on different factors), geographic settings (different places within a certain county/region), and time (a point or a period of time of interest). ${ }^{11}$

To assess overlap between two problems in terms of the population groups, one needs to use a $3 \times 3$ table and assign different population groups/segments into the cells. To assess overlap between two problems in terms of the geographic settings, one needs to use a $3 \times 3$ table and assign different geographic settings into the cells. To assess concurrence between the two problems in terms of their magnitude (at a defined population and place) at a point in time, one needs to use a $3 \times 3$ tool and assign the magnitude of the problems into the cells. The average/medium magnitude to be used for comparison could be national prevalence (for sub-national considerations) or global prevalence (for national considerations). When both problem A and problem B have high magnitude, the need for integrated response is more likely to be higher. This is exemplified in Table 1. To assess epidemiological overlap between two problems in terms of their trend (of magnitude) across time (at a defined population and place), one needs to use a $3 \times 3$ matrix and assign the trends in the magnitude of the problems into the cells (Table 2). A trend-line would be important to assess the presence of overlapping trends. When both problems have an increasing trend, the need for integrated response becomes more likely. The time period for the trend needs to be set based on relevance and availability of data. Trends without a defined pattern may be treated in a different way. Correlational analysis could also be used in such cases.
Correspondence: Tilahun Nigatu Haregu, African Population and Health Research Center, Manga Close, Off Kirawa Road, 10787-00100 Nairobi, Kenya.

Tel: +254.20.400.1000 - Fax: + 254.20.400.1101

E-mail: tilahunigatu@gmail.com

Key words: Healthcare system; Public health problems; Integration.

Received for publication: 15 March 2014.

Revision received: 22 July 2014.

Accepted for publication: 22 July 2014.

This work is licensed under a Creative Commons Attribution 3.0 License (by-nc 3.0).

(C) Copyrigh T.N. Haregu et al., 2015

Licensee PAGEPress, Italy

Healthcare in Low-resource Settings 2015; 3:3260

doi:10.4081/hls.2015.3260

\section{Linkage between the problems}

Information about the inter-relationships between problems is important to inform the content of interventions packages. ${ }^{12}$ The linkage between two problems takes two forms: Risk and Severity. Risk is when the presence of problem A affects the probability of occurrence of problem B and/or vice versa. Severity is when the presence of problem A affects the severity of problem B and/or vice versa.

To assess the linkage between two problems in terms of risk and severity, one needs to compare the risk and severity in the sub-populations with that of the general population. Tool presented in Table 3 summarizes the risk and severity of a problem in the sub-populations, along with a three-point scale, as compared to that of the general population. When data are available, it would be preferable to use quantitative measures of risk and severity to demonstrate actual levels. The greater the risk and severity of the problems in the sub-populations (as compared to the general population), the higher is the need for integrated response.

\section{Co-occurrence of the problems}

Evidence about the magnitude of co-occurrence of two problems in an individual is useful to inform planning and resource allocation. ${ }^{13}$ Co-occurrence of two diseases can be expressed in two forms: Co-morbidity (when there is an index disease) and Multimorbidity (when there is no index disease). ${ }^{14}$ To explore the magnitude of co-occurrence of two problems, one needs to compare the prevalence of each problem among those having the other with that of the general population (for comorbidity); and the prevalence of both diseases in the population to prevalence that would otherwise occur by chance. Tool described in Table 4 summarizes these meas- 
ures.

In situations where actual prevalence values are available, they can be used for the comparison. The greater the prevalence of comorbidity and multimorbidity, the higher the need for integrated response.

\section{Examining similarities between responses}

\section{Define response}

A health system response to a public health problem contains several components at different levels. At upstream (Macro) level are strategic functions including policy making, leadership and governance. At mid-stream (Meso) level are management functions like planning, coordination, resource mobilization etc. At down-stream (Micro) level are operational functions such as service provision, data collection etc. Within each of the elements of the response, several functions and structures are involved. ${ }^{15}$

An effort of integration may involve all or some of these functions/structures. Some processes may require a stronger integration than others. One possible method to establish this is by analysing the similarities between parallel processes (e.g. Treatment of $\mathrm{A}$ and Treatment of B). This is based on the assumption that a higher level of similarity predicts a stronger need for integration. Analysis of similarities between the responses to problem A and Problem B starts with defining the functions of interest that constitutes a response. Depending on the intended focus and type of integration, identify and describe the elements of the response that could be the possible candidates for integration. The scale of the details of these functions would vary based on the level of the health system. An example of list of core functions and their description is presented in Table 5.

\section{Identify comparators}

Once the response functions, the possible candidates for integration, are defined, the next step will be to assess the similarities between the parallel functions. Assessment of similarity between two functions requires comparators - parameters that are used to compare two functions. To identify parameters/attributes of the functions that could be used to compare two processes in order to identify similarities and differences, a list of possible parameters is given in Table 6 .

\section{Rate degree of similarity}

The degree of (relational) similarity is the extent to which a pair of parallel response functions (e.g. prevention of A and prevention of B) shares common parameters/attributes.
Table 1. A 3 X3 matrix for convergence between the problems.

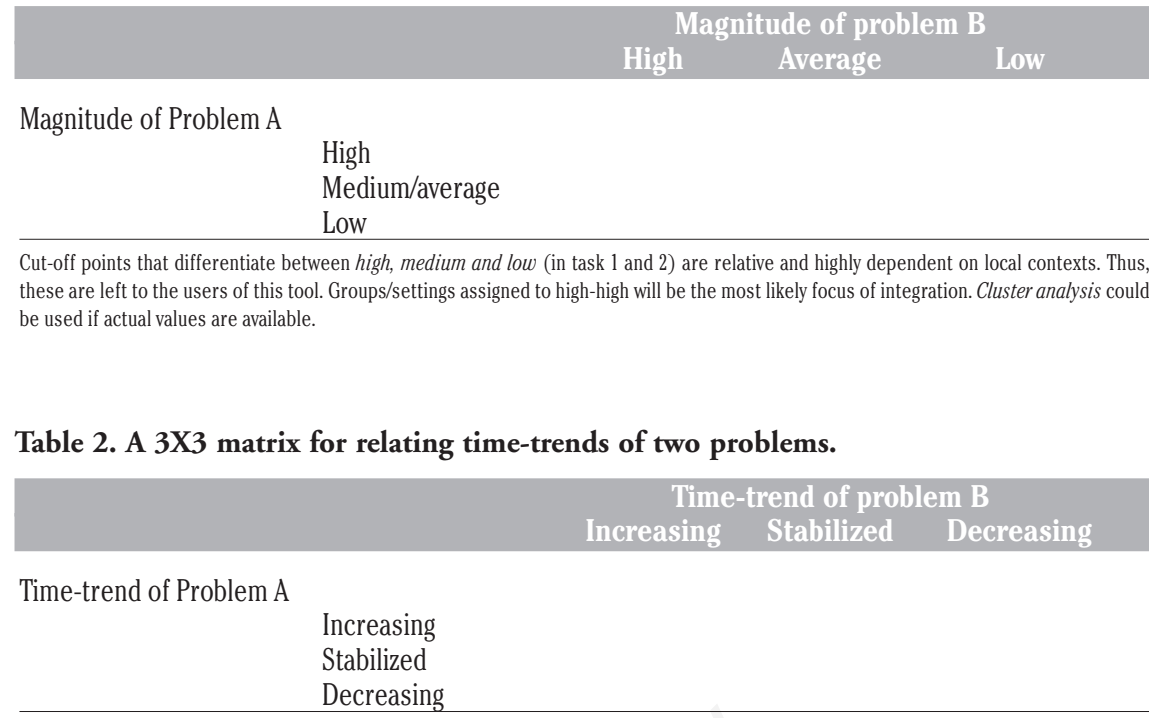

Table 3. Matrix for rating linkage between two problems. Greater Similar Lower

Risk of problem B among A+ as compared to general population Risk of problem $\mathrm{A}$ among $\mathrm{B}+$ as compared to general population

Severity of problem $B$ among A+ compared to general population Severity of problem A among B+ compared to general population

Table 4. Matrix of classifying levels of co-occurrence of two problems. Greater Similar Lower

Prevalence of $A$ among $B+$ as compared to prevalence of $A\left(\mathrm{P}_{A}\right)$ Prevalence of $\mathrm{B}$ among $\mathrm{A}+$ as compared to prevalence of $\mathrm{B}\left(\mathrm{P}_{\mathrm{B}}\right)$ Prevalence of $\mathrm{AB}$ in general population as compared to $\left(\mathrm{P}_{\mathrm{A}} * \mathrm{P}_{\mathrm{B}}\right)$

Table 5. List of major functions that constitute response to health problems.

\begin{tabular}{lll}
\hline Categories & Functions & Description of the functions \\
Policy & Leadership & High level political commitment \\
& Policy advising & Providing inputs for policy making \\
& Policy making & Formulation/approval of policies \\
Governance & Overseeing policy implementation processes \\
Program & Prevention & Measures taken to prevent disease \\
& Treatment & Services provided to control/treat disease \\
& Care and support & Services provided to improve quality of life \\
& System strengthening & Interventions that improve system capacity \\
\hline Management & Planning & Strategic and annual planning \\
& Implementation & Overseeing implementation of programs \\
& Resource mobilization & Securing resources needed for programs \\
& Multisectoral coordination & Coordination of multiple actors/sectors \\
Strategic information & Patient monitoring & Monitoring the progress of patients \\
& Disease monitoring & Monitoring of disease/epidemic patterns \\
& Program M\&E & Monitoring and evaluation of programs \\
Dissemination & Communication of findings of M\&E \\
\hline
\end{tabular}


The most appropriate and applicable set of parameters should be used for the rating. The rating scale may vary from dichotomous scale to a higher point Likert scales. Using a selected set of parameters, one should rate the degree/strength of similarities between a pair of parallel functions. A sample template for rating the similarity between program related functions of problem A and Problem B is given in Table 7.

\section{Determine importance of similari- ties}

In addition to the degree of similarity, the relative importance of similarity is also essential. The importance of the similarities between a pair of parallel functions can be viewed from four major perspectives: policy the strategic importance of the similarity for policy purpose; managerial - the importance of the similarity for decision making; economic the importance of the similarity in efficient use of resources; and practical - the importance of the similarity for program implementation. To determine the relative importance of the similarities between a pair of parallel functions by considering the policy, management, economical, and practice perspectives one should follow Table 8.

\section{Scanning the environment for integration}

After establishing the need for integration (section I) and identifying candidate functions/structures for integration (section II), the third phase is assessing whether the environment is enabling/conducive for integration. This is conducted using environmental scanning. In principle, three components of the environment need to be considered: Internal (Staffs, Managers, Organizational set up), Task-related (patients, competitors i.e. other actors, partners, donors, pressure groups), and External (Political, Economic, Socio-cultural and Technological factors). From the perspective of integration, the following themes are important.

\section{Motivation for integration}

Interest among managers and staffs (of Unit A and Unit B) to integrate the relevant functions/structures and operate in an integrated approach. To assess whether policy makers, managers and staffs of unit A and unit B are interested to integrate the respective functions and thereby operate in an integrated approach one should follow Table 9 .

\section{Capacity for integration}

Capacity to integrate (for managers) and capacity to operate in an integrated approach
Table 6. List of potential parameters that may be used to assess similarity.

\begin{tabular}{ll} 
Parameters & Descriptions \\
Operational characteristics & Nature and technical complexity \\
Timing of the functions & Time and frequency (when and how often) \\
\hline Actors/performers & The skills/expertise/speciality required \\
Methods/tools & Models and approaches used \\
\hline Targets/users & The characteristics of the customers/users \\
Results/outputs & The attributes of the end products \\
\hline Input requirements & Monetary and non-monetary requirements \\
\hline Levels in the system & Levels of health system where the functions happen \\
\hline Lines of accountability & Command and communication chains \\
Monitoring modalities & Monitoring requirements (formats, schedules, etc.) \\
\hline Priority and interests & Accorded priorities and vested interests \\
\hline
\end{tabular}

Table 7. Matrix for rating degree of similarity of parallel functions.

Pairs of parallel functions
(these are examples only, add more to this list) Low $\quad$ Medium

Prevention (of $A$ and $B$ )

Treatment (of $A$ and $B$ )

Care \& support (of A and B)

Health system strengthening (of A and B)

Table 8. Matrix for rating relative importance of similarity between parallel functions.

Similarity between
(these are examples only)
Prevention (of A and B)
Treatment (of A and B)
Care and support (of A and B)
Health system strengthening (of A and B)
At the end of this section, an initial short-list of possible candidates (for integration) of response functions would be reached. Though higher
degrees of similarity and higher relative importance of the similarity could be the mainstay of the selection, this will also depend on judgement
by the responsible body.

Table 9. Matrix for rating levels of motivation towards and capacity for integration.

$\begin{array}{ccc}\text { Levels of motivation } & \text { Ledium High }\end{array}$

Policy makers

Managers

Practitioners

Levels of capacity

Managerial capacity

Technical capacity

Institutional capacity

Table 10. Matrix for rating levels of acceptability of integration by end users.

$\begin{array}{ccc}\text { Find users } & \text { Levels of acceptability } \\ & \text { Low } & \text { Medium }\end{array}$

Service users/customers (e.g. patients)

Funding agencies (donors)

Governing bodies (including government) 
(for staffs and institution/infrastructure). To assess the capacity (managerial, technical and institutional) to integrate the functions and operate in an integrated approach one should refer to Table 9 .

\section{Acceptability of integration}

The extent to which the integrated approach is acceptable to the end users (patients, donors, governments) of the processes or the arrangements. To assess whether an integrate approach is acceptable to end users of the functions one should follow Table 10.

\section{Influences on integration}

The effects (reactions) of important stakeholders and their activities on integration process. Influences may be negative, neutral or positive. To assess the possible reactions of other important stakeholders towards the integrated approach one should refer to Table 11.

\section{Implications of integration}

The possible effects (impacts) of the integration on important stakeholders and their business. This may also be positive, neutral or negative. Assessing how the integration of the functions/structures might affect other important stakeholders is described in Table 11.

\section{Repackaging evidence for inte- gration}

All the preceding sections of this tool were designed for generating important evidence about the need for integration, identifying the appropriate candidate functions/units for integration and assessing the conduciveness of health system environment for integration. The evidence generated needs to be repackaged in a form that can better inform decisions related to integration. A matrix of four major elements of evidence communication should include: purpose, audience, content/message, method.

\section{The audience (Who)}

Integration may mean different things for different people. Policy makers, managers, healthcare providers, patients, and researchers have different views about integration. Repackaging evidence of integration needs to take into account these views and interests. The task of this section is to clearly state the target audience, their views, and their interests in relation to integration.

\section{The purpose (Why)}

Repackaging of integration related evidence should be targeted towards achieving a clearly defined purpose. The purpose is usually instrumental - for practical applications. In some instances, however, it may be symbolic - to confirm decisions, policies and practices. The task of this section is to clearly state the purpose(s) of the communication of evidence about integration.

\section{The content (What)}

What needs to be included in the communi- cation package depends on the purpose and the audience of the communication. The task of this section is to prepare the content of communication product - the knowledge/evidence that is going to be communicated.

\section{The method (How)}

The method of communication may be selected based on knowledge about the interests of the audience. It may be in the form of printed materials, electronic materials, audiovisuals, conference presentations, etc. The task of this section is to decide on the method of communication and appropriate communication product.

\section{Managing integration}

Once the evidence about integration is effectively communicated, responsible bodies are expected to make decision about the integration. The translation of that decision in to action should be systematic, with steps involving planning, implementation, Monitoring and Evaluation.

Table 11. Matrix for classifying anticipated reactions of stakeholders and impacts of integration on them.

$\begin{array}{ccc}\text { Important stakeholders } & \text { Anticipated reactions } & \text { Negative } \\ \text { Neutral } & \text { Positive }\end{array}$

Stakeholder 1

Stakeholder 2

(add rows for more stakeholders)

Stakeholder 1

Stakeholder 2

(add rows for more stakeholders)

Table 12. The ten levels of integration.

Levels Communication Consultation Coherence Consensus Coordination Cooperation Collaboration Co-location Coalition Combination
of integration

Baseline level

Target level

Table 13. Major constructs for evaluation of integration.

Indicators for Before integration After integration Change

Level of integration

Systems' performance

Cost performance units

Objectives of integration

Goals of health system 


\section{Planning integration}

Integration should be a well-planned process. Integration planning needs to consider the parts and the parties that are going to be integrated. Depending on its extent, integration planning may address a range of tasks: i) select the foci of integration (units/functions that are going to be integrated), which may include functions/structures relevant to policy, institutional arrangement, management, program, and information; ii) formulate the goals/objective of the integration; iii) determine baseline (the existing) and the target (the desired level) of integration for each foci of integration (Table 12); iv) identify strategies/mechanisms to be used to achieve objective of the integration; v) estimate the cost/resources required for implementing the strategies; vi) weigh the benefits and risks that might be associated with the integration.

Once this is done, one should define the key elements of integration plan and prepare the plan.

\section{Implementing integration}

This step is about the application of the integration plan in to action. It involves operationalization of integration plan in to implementation plan and carrying out activities as per the implementation plan. The implementation of integration plan, therefore, involves: i) operationalization (i.e. deciding who will do what and when); ii) implementation (i.e. translating the implementation plan in to action); iii) coordination (i.e. synchronizing activities and actors); iv) supervision (i.e. supervising and taking corrective action); v) monitoring (i.e. measuring progress and comparing against the plan).

\section{Evaluating integration}

As any other performance improvement initiative, integration should be evaluated (Table 13). The key constructs that are usually important in the evaluation of integration are: configuration (whose objective is to describe the alignment of the processes before and after integration and explain the differences in the integration architecture); synergy [aimed at measuring performance of the integrated architecture (after integration) and compare it with the sum of performance of the units (before integration)]; efficiency (which calculates the unit cost per performance units before and after the integration and describe the differences); effectiveness [whose aim is to determine the level of achievement of the stated objectives of the integration (as stated in the integration plan)]; impact (aimed at determining the difference between the level of achievements of the objectives of the health system before and after the integration).

\section{Conclusions}

The proposed generic tool is developed based on the existing evidence relevant to the integration of responses to major public health problems. It has laid out the basic processes and sub-processes that need to be undertaken in the process of integrating system level responses in a systematic manner. It provides guidance for a comprehensive, evidence-based and step-wise approach to integration. As it includes the generation, synthesis, and utilization of evidence in its steps, it can suit situations where evidence relevant to integration is yet to be generated. However, this tool has undergone only conceptual and content validation. Further studies are needed to evaluate how the tool can be best streamlined into various health systems.

\section{References}

1. Suter E, Oelke ND, Adair CE, Armitage GD. Ten key principles for successful health systems integration. Healthcare Q 2009;13:16-23.

2. Shigayeva A, Atun R, McKee M, Coker R. Health systems, communicable diseases and integration. Health Policy Plann 2010;25(Suppl.1):4-20.

3. Miranda JJ, Kinra S, Casas JP, et al. Noncommunicable diseases in low- and middle-income countries: context, determinants and health policy. Trop Med Int Health 2008;13:1225-34.
4. Armitage GD, Suter E, Oelke ND, Adair CE. Health systems integration: state of the evidence. Int J Integr Care 2009;9:e82.

5. Haregu TN, Setswe G, Elliott J, Oldenburg B. Developing an action model for integration of health system response to HIV/AIDS and noncommunicable diseases (NCDs) in developing countries. Glob J Health Sci 2013;6:9-22.

6. Budetti PP, Shortell SM, Waters TM, et al. Physician and health system integration. Health Affair 2002;21:203-10.

7. Russell E, Johnson B, Larsen H, et al. Health systems in context: a systematic review of the integration of the social determinants of health within health systems frameworks. Rev Panam Salud Publ 2013;34:461-7.

8. Jackson SF, Birn AE, Fawcett SB, et al. Synergy for health equity: integrating health promotion and social determinants of health approaches in and beyond the Americas. Rev Panam Salud Publ 2013;34:473-80.

9. Evans JM. Health systems integration: competing or shared mental models? Int J of Integr Care 2014;14:e028.

10. Tsasis P, Evans JM, Forrest D, Jones RK. Outcome mapping for health system integration. J Multidisc Healthc 2013;6:99-107.

11. Sword W. A socio-ecological approach to understanding barriers to prenatal care for women of low income. J Adv Nurs 1999;29:1170-7.

12. Govindasamy D, Kranzer K, van Schaik N, et al. Linkage to HIV, TB and non-communicable disease care from a mobile testing unit in Cape Town, South Africa. PloS One 2013;8:e80017.

13. Shwartz M, Iezzoni LI, Moskowitz MA, et al. The importance of comorbidities in explaining differences in patient costs. Med Care 1996;34:767-82.

14. Valderas JM, Starfield B, Sibbald B, et al. Defining comorbidity: implications for understanding health and health services. Ann Fam Med 2009;7:357-63.

15. Murray CJ, Frenk J. A framework for assessing the performance of health systems. B World Health Organ 2000;78:71731 . 